\title{
Finite-difference modelling of wavefield constituents
}

\section{Journal Article}

Author(s):

Robertsson, Johan O.A.; van Manen, Dirk-Jan; Schmelzbach, Cédric (D; van Renterghem, Cédéric; Amundsen, Lasse

Publication date:

2015-11-01

Permanent link:

https://doi.org/10.3929/ethz-b-000109134

Rights / license:

In Copyright - Non-Commercial Use Permitted

Originally published in:

Geophysical Journal International 203(2), https://doi.org/10.1093/gji/ggv379 


\title{
Finite-difference modelling of wavefield constituents
}

\author{
Johan O.A. Robertsson, ${ }^{1}$ Dirk-Jan van Manen, ${ }^{1}$ Cedric Schmelzbach, ${ }^{1}$ Cederic Van \\ Renterghem ${ }^{1}$ and Lasse Amundsen ${ }^{2}$ \\ ${ }^{1}$ ETH Zürich, Institute of Geophysics, Zürich, Switzerland.E-mail: johan.robertsson@erdw.ethz.ch \\ ${ }^{2}$ University of Science and Technology (NTNU) and Statoil Research Centre, Trondheim, Norway
}

Accepted 2015 September 8. Received 2015 September 8; in original form 2015 June 30

\begin{abstract}
SUMMAR Y
The finite-difference method is among the most popular methods for modelling seismic wave propagation. Although the method has enjoyed huge success for its ability to produce full wavefield seismograms in complex models, it has one major limitation which is of critical importance for many modelling applications; to naturally output up- and downgoing and $P$ - and $S$-wave constituents of synthesized seismograms. In this paper, we show how such wavefield constituents can be isolated in finite-difference-computed synthetics in complex models with high numerical precision by means of a simple algorithm. The description focuses on up- and downgoing and $P$ - and $S$-wave separation of data generated using an isotropic elastic finitedifference modelling method. However, the same principles can also be applied to acoustic, electromagnetic and other wave equations.
\end{abstract}

Key words: Numerical solutions; Theoretical seismology; Wave scattering and diffraction; Wave propagation.

\section{INTRODUCTION}

Modelling of seismic wave propagation plays a key role in almost every aspect of exploration seismology. It is used to provide an understanding of how waves propagate in a complex heterogeneous real Earth. Modelling is also used for survey design (Regone 2007), imaging (Vasconcelos 2013), inversion (Brenders \& Pratt 2007) and seismic data processing (e.g. to attenuate multiples or noise; Amundsen \& Robertsson 2014). Finally, generating synthetic data sets for research has proven extremely valuable in developing and testing new data processing algorithms (Özbek et al. 2010).

A wide range of modelling methods is available to generate synthetic data. A particularly popular method in the exploration seismic community is the finite-difference (FD) method (e.g. Virieux 1986; Levander 1988). The FD method is relatively straightforward to implement and enables the computation of seismograms that include the full wavefield propagating in complex heterogeneous models that can be acoustic, elastic, viscoelastic, anisotropic or poroelastic in entire or parts of a model (Moczo et al. 2007; Robertsson \& Blanch 2014). Although the method is widely used, it also has some drawbacks compared to other methods. In this paper, we show how to eliminate one of these drawbacks, namely the perceived inability of the method to naturally output decomposed wavefield constituents such as up- and downgoing wavefields as well as their $P$ - and $S$-wave constituents. Such synthetic data are essential for instance when developing and testing methods for multiple attenuation (Dragoset \& Jericevic 1998; Amundsen 2001), noise attenuation (Halliday et al. 2010) or deghosting (Robertsson \& Amundsen 2014). To date, one of the few full wavefield modelling techniques that naturally computes such wavefield constituents is the reflectiv- ity method (Kennett 1983). In this paper, we show how FD methods for the elastic wave equation also can output such wavefield constituents. However, the same methodology will also be applicable for the solution of both the acoustic wave equation as well as the electromagnetic wave equation. Our FD methodology operates in the time-space domain and therefore, as opposed to the reflectivity method for instance, we avoid potentially cumbersome transformations between the frequency-slowness and time-space domains.

Frequency-wavenumber and frequency-space domain filters that separate elastic wavefield data into up- and downgoing waves and $P$ and $S$ waves have been presented before (e.g. Fokkema $\&$ van den Berg 1993; Amundsen et al. 2000). In principle, such filters can of course also be applied to the output of FD simulations. However, these filters are difficult to implement with high precision, particularly close to grazing angles for instance. Recently it has been recognized that proper injection of multicomponent seismic data on FD grids propagates recorded super-imposed up- and downgoing wavefields with their correct propagation directions (e.g. Blanch 2012; Ravasi \& Curtis 2013; Vasconcelos 2013; Amundsen \& Robertsson 2014). However, the full implication of these results for FD modelling has not been realized so far. We base our methodology on the so-called FD-injection technique (e.g. Robertsson \& Chapman 2000) to separate the synthesized data into its up- and downgoing constituents as outlined by Amundsen \& Robertsson (2014). Of particular importance is the fact that since we are using the same FD method (and simulation parameters) to separate the data as was used to generate the data, the separation into wavefield constituents will result in a decomposition that can be close to machine precision accuracy independent of incidence angles and other complexities of the wavefield. 

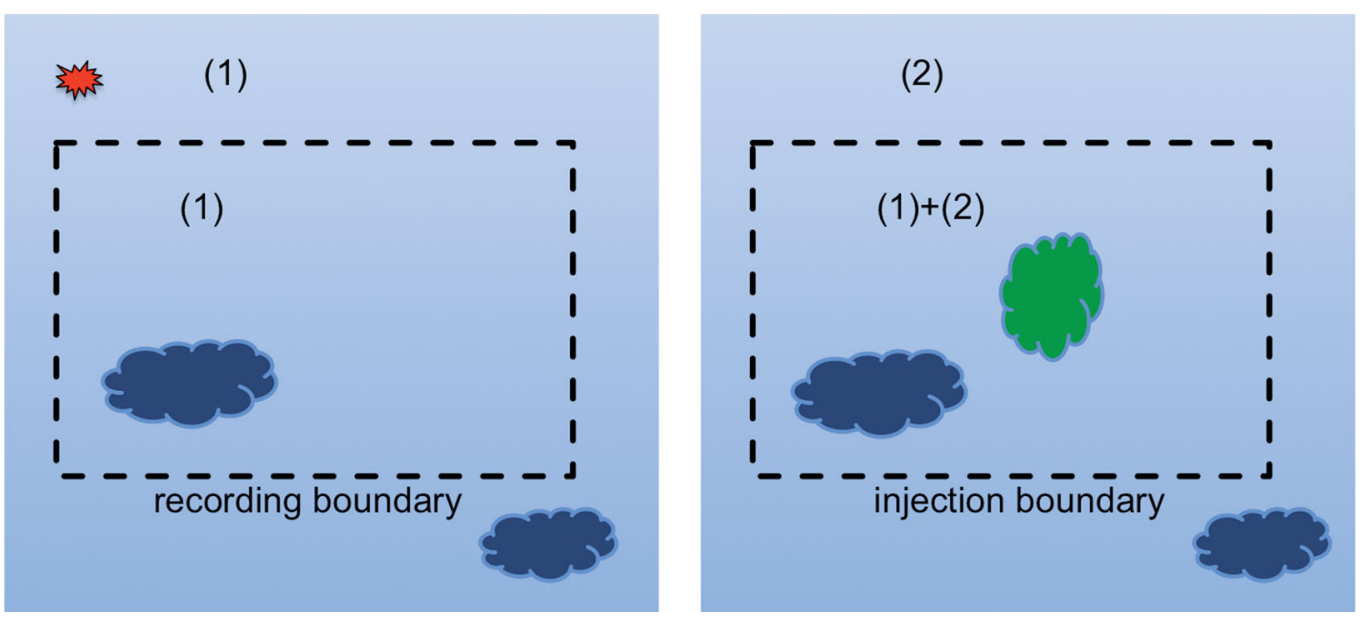

Figure 1. Configuration of FD simulations in the conventional application of FD-injection (Robertsson \& Chapman 2000). Left: initial computation when recording the injection wavefields along the recording/injection boundary. Right: wavefield injection recomputation after modifying the model inside the injection surface. Wavefield constituent (1) represents the original unperturbed wavefield whereas wavefield constituent (2) represents the perturbed part of the wavefield caused by the introduction of the green scatterer in the wavefield injection computation.

We begin by recapitulating the FD-injection technique (Robertsson \& Chapman 2000). Next we discuss how to separate wavefield constituents into up- and downgoing as well as $P$ and $S$ constituents by means of FD-injection. Finally, we demonstrate the methodology through a simple example.

\section{FD-IN JECTION}

Robertsson \& Chapman (2000) describe a technique, referred to as FD-injection, for recomputing the response of FD solutions after local model alterations. The method is closely related to the method for source wavefield injection described by Alterman \& Karal (1968) and relies on two fundamental observations:

(1) The principle of superposition and the fact that the wave equation is linear. If two wavefield constituents are present on an FD grid and if one of them is known, the other wavefield constituent is simply obtained by subtracting the known wavefield constituent from the total wavefield.

(2) The observation that FD stencils are compact in space.

In Fig. 1, we illustrate the different steps of the FD-injection methodology. Initially an FD simulation is carried out on an unperturbed model (Fig. 1, left). During this simulation the wavefield is recorded along a closed (transparent) surface around a target of interest. To be more precise, the wavefield is recorded on as many neighbouring grid points to the surface as the FD stencil used is wide (in space). In a second step, the model is altered inside the closed surface (illustrated by the green scatterer in the right part of Fig. 1). Next we wish to recompute the wavefield after the model alteration. This can be done by injecting the wavefield from the first simulation on the same surface but now on the perturbed model. Since the wavefield to be injected is known this can be added and subtracted as appropriate for the update of points along the injection surface for the parts of the spatial stencils that intersect the injection surface (subtract the injection wavefield at the appropriate points when updating points in the immediate exterior of the injection surface and adding the injection wavefield at the appropriate points when updating points in the immediate interior of the injection surface). As a consequence we will observe an apparent discontinuity across the injection surface. Inside the surface both the injected wavefield [identical to the initial computation in the unperturbed model and denoted by (1) in Fig. 1] as well as the perturbed wavefield [difference in wavefields before and after the model alteration and denoted by (2) in Fig. 1] are present and superimposed. Outside the injection surface only the perturbed wavefield is present, appearing to leak through the injection surface (note that if the model is left unperturbed in the second simulation, the wavefield outside the injection surface will be as close to zero as machine precision allows).

The FD-injection method was originally proposed benefitting from the fact that in the second simulation when the wavefield is recomputed, the model can be made much smaller encompassing the region of change and major nearby scatterers to recompute the wavefield after model alterations. Robertsson et al. (2000) showed how this results in substantial computational saving in a time-lapse seismic modelling application. Borisov \& Singh (2013) use the FDinjection method to significantly reduce the computational cost in target-oriented elastic full waveform inversion.

The FD-injection technique described by Robertsson \& Chapman (2000) relies on the recording and injection of wavefields on a closed surface on an FD grid. Amundsen \& Robertsson (2014) discuss the consequence of using an open surface (e.g. a single horizontal segment) while injecting wavefields on different models. In particular, they discuss injecting wavefields on a homogeneous model with the material properties corresponding to those of the location of the original recording surface (assumed to be uniformly homogeneous) as illustrated in Fig. 2. The left part of Fig. 2 shows the original recording datum (corresponding to an open surface), whereas the right part shows the injection surface on a different model with the (homogeneous) material properties of the recording datum. The wavefield that is injected in the model shown in the right part of Fig. 2 comprises two parts. First, normal to the surface (the inwards direction if the surface would have been closed), only wavefields radiate from the injection surface that are propagating inwards. These wavefields are injected with correct polarity. Second, in the opposite direction to the normal, only wavefields radiate from the injection surface that are propagating outwards but with opposite polarity. The injected wavefields will strictly propagate away from the injection surface on the homogeneous model, as after injection the propagating wavefields cannot change direction to cross the injection surface. As a result the injected wavefield will be separated into up- and downgoing 


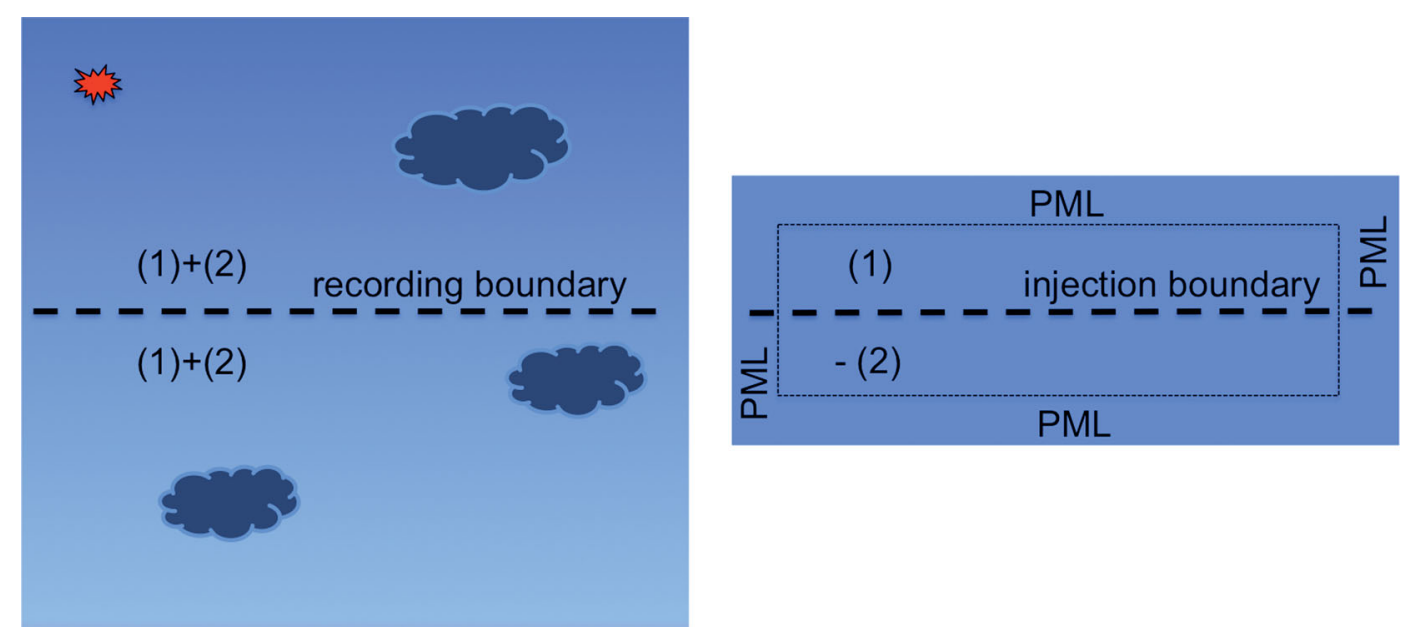

Figure 2. Configuration of FD simulations in the application of FD-injection (Robertsson \& Chapman 2000) proposed in this paper. Left: initial computation when recording the injection wavefields along the injection boundary. Right: wavefield injection recomputation on a homogeneous model to separate the wavefields (Amundsen \& Robertsson 2014). Wavefield constituent (1) represents the upgoing wavefield whereas wavefield constituent (2) represents the downgoing wavefield.

constituents on opposite sides of the injection surface [illustrated in Fig. 2 where wavefield constituent (1) represents the upgoing wavefield and wavefield constituent (2) represents the downgoing wavefield]. This result is fully consistent with the observations presented above where on a closed surface and an unperturbed model, these outward injected wavefield constituents would destructively interfere with their propagating counterparts that have originally been injected as ingoing wavefields before propagating on the unperturbed model. Furthermore, these intuitive results are not entirely surprising as Masson et al. (2014) have established the equivalence between the FD-injection methodology and time-reversal mirrors for tomographic imaging. The results presented by Amundsen \& Robertsson (2014) are therefore conceptually equivalent with similar observations presented by Blanch (2012), Ravasi \& Curtis (2013) and Vasconcelos (2013) where wavefields are introduced by injecting recordings as 'multiple point sources' using representation theorems (Fokkema \& van den Berg 1993).

\section{Separation of FD modelled wavefields into up- and downgoing constituents}

When using an FD method to solve a partial differential equation, a numerical error (NE) is introduced such that the solution computed corresponds to a certain 'finite difference equation' (FDE) which is different to the solution of the desired partial differential equation (PDE): FDE = PDE + NE (Pletcher et al. 2012). This general statement is true for any linear partial differential equation including the wave equation. Whereas Amundsen \& Robertsson (2014) discuss the general use of FD-injection for wavefield separation, this paper is concerned with the application of the same methodology applied to synthetic data generated using the same underlying FD engine. A subtle but critical point of this paper is the realization that if (and only if) we use the method of FD-injection to separate FD-generated synthetics into their up- and downgoing parts as described by Amundsen \& Robertsson (2014), we exactly generate the up- and downgoing constituents corresponding to the FDE to within machine precision.

In the following we limit the discussion to the case where the recording datum is located in a homogeneous part of the model (we briefly address the general case of the recording datum intersecting a heterogeneous part of the model in the Discussion section). As described above and as illustrated in Fig. 2 the cost of the methodology is proportional to two FD simulations. However, the model in the second FD simulation can be much smaller than in the original simulation; the FD model should only be large enough to encompass the recording datum and should be homogeneous with the material properties of the location of the recording datum in the original simulation. As described above, FD-injection will automatically separate the up- and downgoing wavefields radiating on opposite sides of the injection boundary. In principle, the technique works to within machine precision of the computations. However, boundary reflections from the absorbing boundaries in the two simulations are likely to dominate the error. Nevertheless, in the second simulation where the wavefields are separated, we benefit from the fact that the model is homogeneous and that perfectly matched layer (PML) absorbing boundary conditions (Komatitsch \& Martin 2007) can be made working to an extremely high degree of accuracy in such a case. Also, by letting the edges of the open injection surface partly intersect with the PML boundary, edge diffractions are avoided without having a significant effect on the wavefield separated data in the vicinity of the injection location. This is because PML's attenuate waves that propagate perpendicular to the PML boundary whereas waves propagating parallel to the boundary are not affected.

\section{Separation of up/down separated modelled wavefields into $P$ - and $S$-wave constituents}

In modelling of elastic wavefields, it is often also of interest not only to know the up- and downgoing constituents but also to know the parts that constitute $P$ waves and the parts that constitute $S$ waves (Yan \& Sava 2008). In the following we limit the discussion to the isotropic elastic case where the separation of $P$ and $S$ waves is straightforward; we separate the wavefield into a curl-free and a divergence-free part. In a homogeneous, isotropic, source-free medium the elastic wave equation in terms of particle velocity $\bar{v}$ is given by:

$\frac{\partial^{2} \bar{v}}{\partial t^{2}}=\alpha^{2} \nabla(\nabla \cdot \bar{v})-\beta^{2} \nabla \times(\nabla \times \bar{v})$, 


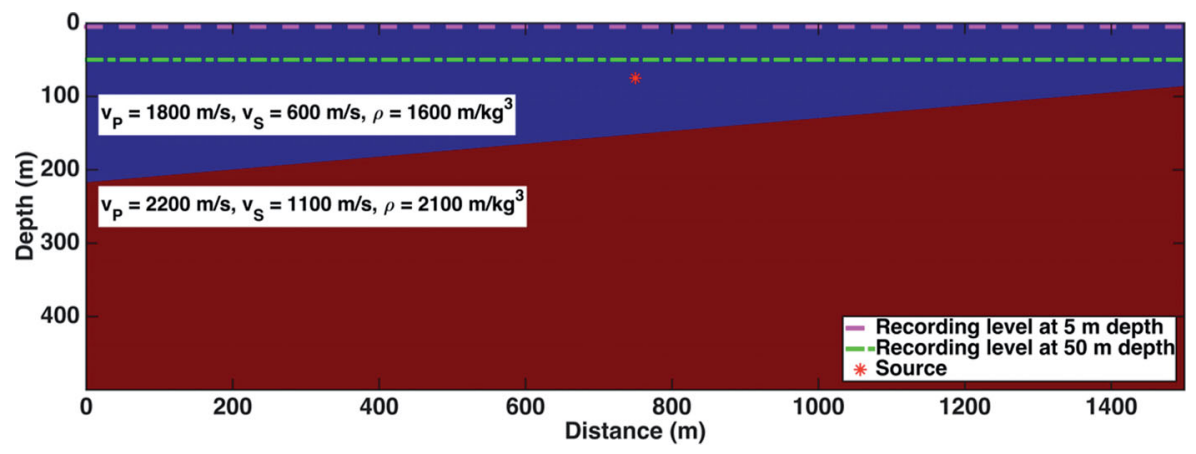

Figure 3. 2-D model used in the example. The model is bounded by a free surface on the top. The top layer has a $P$-wave velocity of $1800 \mathrm{~m} \mathrm{~s}{ }^{-1}$, an $S$-wave velocity of $600 \mathrm{~m} \mathrm{~s}^{-1}$ and a mass density of $1600 \mathrm{~kg} \mathrm{~m}^{-3}$. The lower interface of the upper layer is planar with a dip of $5^{\circ}$. The bottom layer has a $P$-wave velocity of $2200 \mathrm{~m} \mathrm{~s}^{-1}$, an $S$-wave velocity of $1100 \mathrm{~m} \mathrm{~s}^{-1}$ and a mass density of $2100 \mathrm{~kg} \mathrm{~m}^{-3}$.
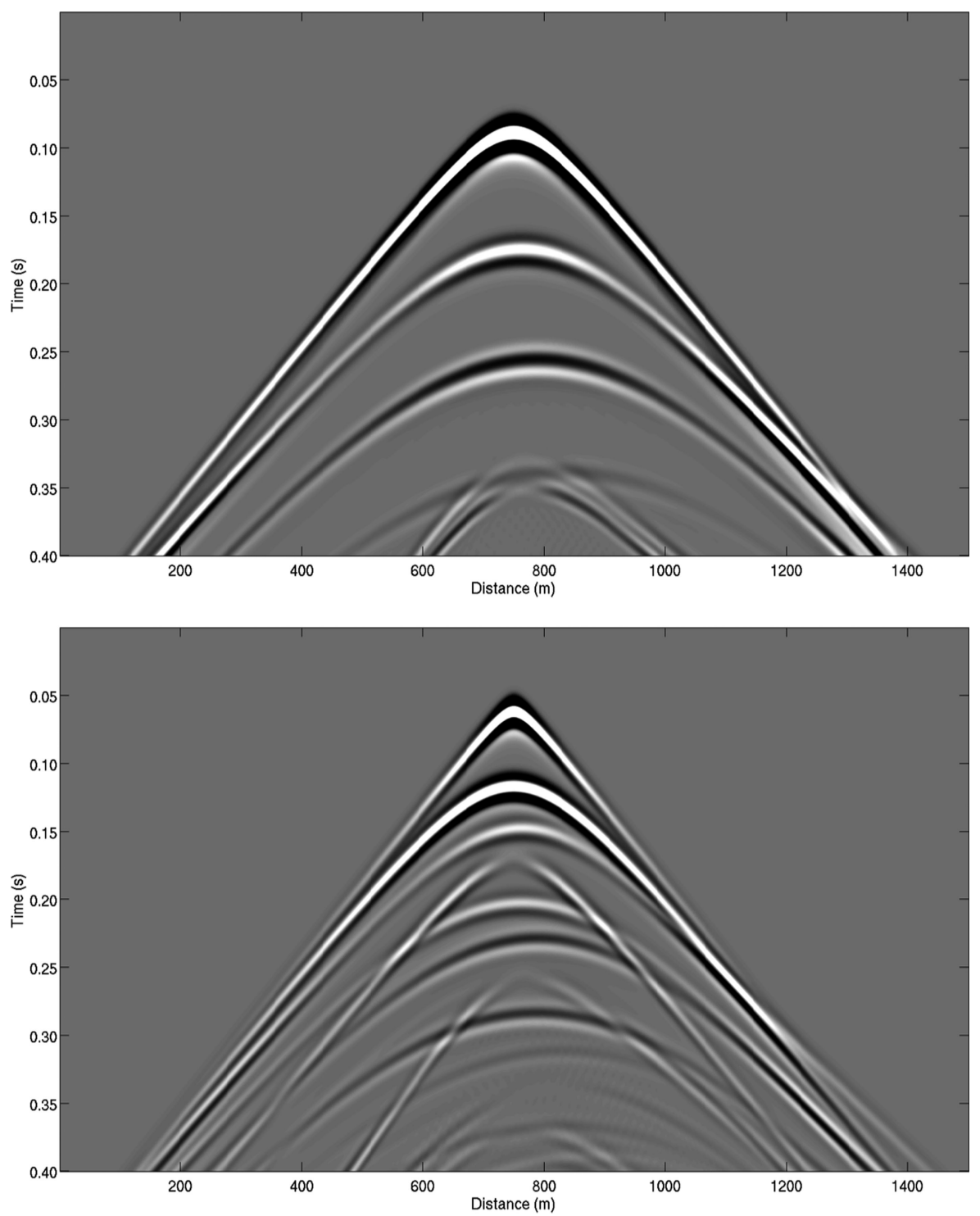

Figure 4. Vertical component of particle velocity recorded $5 \mathrm{~m}$ below the free surface (top) and $50 \mathrm{~m}$ below the free surface (bottom).

where $\alpha$ is the $P$-wave velocity of the medium and $\beta$ is the $S$-wave velocity (Chapman 2004). In a homogeneous medium, separating $P$ and $S$ waves is straightforward as eq. (1) decouples into two wave equations for $P$ and $S$ waves, respectively. After injecting the wavefield to separate up- and downgoing waves as described above, the parts of the injected particle velocity field that correspond to $P$ and $S$ waves are obtained respectively by applying the appropriate spatial operator as well as integrating twice in time:

$$
\bar{v}_{\alpha}=\alpha^{2} \iint \nabla(\nabla \cdot \bar{v}) d t^{2},
$$



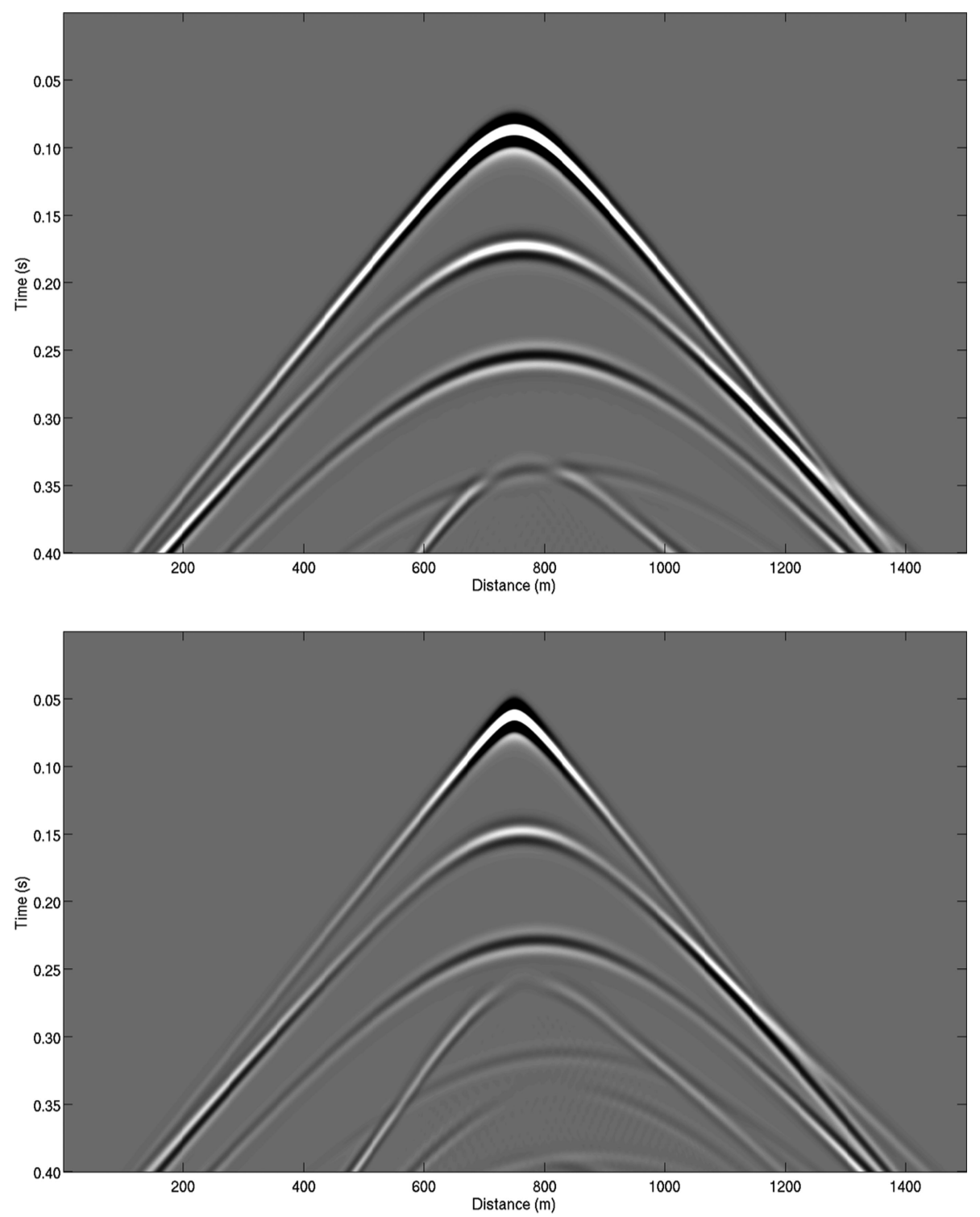

Figure 5. Upgoing vertical component of particle velocity $5 \mathrm{~m}$ below the free surface (top) and $50 \mathrm{~m}$ below the free surface (bottom).

and

$\bar{v}_{\beta}=\beta^{2} \iint \nabla \times(\nabla \times \bar{v}) d t^{2}$.

Generalizations to anisotropic media are described by Dellinger \& Etgen (1990) and Yan \& Sava (2009).

Since the $P / S$ separation step is applied to the up/down separated data that result from the injection of the FD generated synthetics on a model with uniform medium properties corresponding to those at the recording datum, major unconformities just above or below the recording datum, such as the Earth's free surface or a fluid-solid interface (e.g. the seafloor), do not present a problem.

Note that whereas the up/down-separation is accurate to within machine precision as long as the same FD parameters (time- and spatial discretization) are used, the accuracy of the $P / S$ separation will only be as accurate as the operators used to approximate eqs (2) and (3).

\section{EXA M P L E}

We demonstrate the methodology with a simple 2-D example. The FD method is a conventional explicit staggered grid solution to the system of first-order partial differential equations in terms of particle velocities and stresses describing elastic wave propagation (Robertsson \& Blanch 2014). The method is second-order accurate in time and fourth-order accurate in space.

The model shown in Fig. 3 is bounded by a free surface on the top (at $z=0 \mathrm{~m}$ ) and consists of two layers. The top layer has a $P$-wave velocity of $1800 \mathrm{~m} \mathrm{~s}^{-1}$, an $S$-wave velocity of $600 \mathrm{~m} \mathrm{~s}^{-1}$ and a mass density of $1600 \mathrm{~kg} \mathrm{~m}^{-3}$. The lower interface of the upper layer is planar with a dip of 5 degrees. The bottom layer has a $P$-wave velocity of $2200 \mathrm{~m} \mathrm{~s}^{-1}$, an $S$-wave velocity of $1100 \mathrm{~m} \mathrm{~s}^{-1}$ and a mass density of $2100 \mathrm{~kg} \mathrm{~m}^{-3}$. An explosive Ricker wavelet point source with a $50 \mathrm{~Hz}$ centre-frequency is located at $x=750 \mathrm{~m}$ and $z=75 \mathrm{~m}$. Data are output, decomposed and compared at two different depth levels: at $z=5 \mathrm{~m}$ and $z=50 \mathrm{~m}$. We show results in terms of vertical component of particle velocity only. Results for 

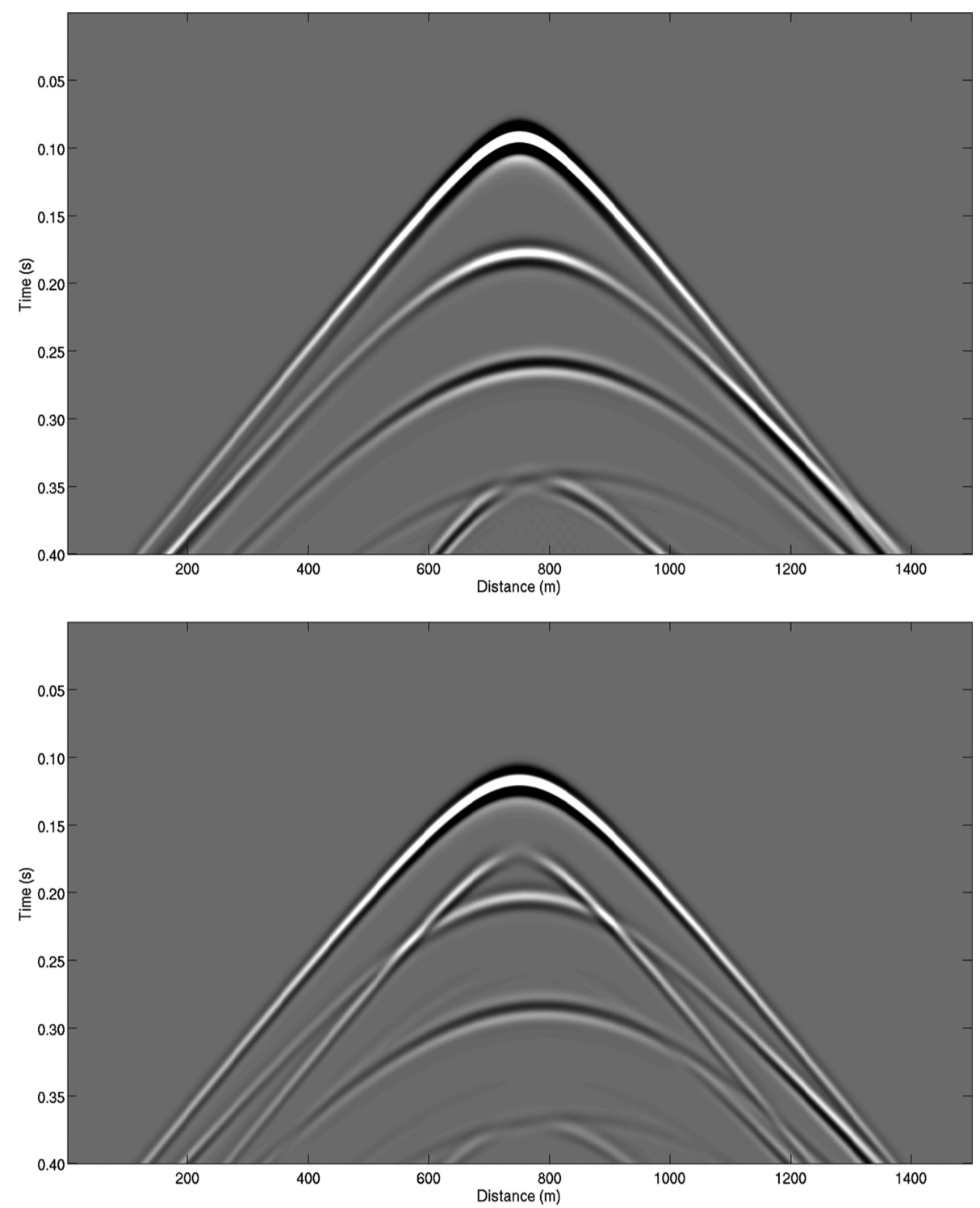

Figure 6. Downgoing vertical component of particle velocity $5 \mathrm{~m}$ below the free surface (top) and $50 \mathrm{~m}$ below the free surface (bottom).

the horizontal component of particle velocity are produced in an analogous fashion.

Fig. 4 shows the total wavefield at the two recording levels. At the shallow recording level $5 \mathrm{~m}$ below the free surface (top plot), upand downgoing waves as well as their $P$ - and $S$-wave constituents overlap throughout the seismograms as waves reflect and convert at the free surface. At the lower recording level $(50 \mathrm{~m}$ below the free surface; bottom plot), up- and downgoing waves as well as $P$ - and $S$-wave constituents no longer overlap completely.

Figs 5 and 6 show the up- and downgoing wavefield constituents at the two recording levels. In Fig. 5, we show the upgoing wavefield constituents $5 \mathrm{~m}$ (top) and $50 \mathrm{~m}$ (bottom) below the free surface and in Fig. 6 we show the analogous results for the downgoing wavefield. We can clearly identify corresponding arrivals in the one-way wavefields at the two depth levels but with expected time shifts (depending on propagation angles and whether they are $P$ or $S$ waves).

Fig. 7 compares three traces of the upgoing wavefield displayed in Fig. 5 against a reference solution where we have computed the wavefield using the same FD method in the same model but without the free surface present. In such a model, we expect all waves to be upgoing and that no arrivals that interact multiple times with the interface (i.e. surface-related multiples) will be present. We see an excellent match of the direct wave and the reflections from the interface. Again, since the same FD simulation parameters are used during the injection calculation as in the original computation, the wavefield decomposition is accurate to within machine precision. However, as expected the reference solution does not contain the multiple reflected upgoing events present in our FD solution on the full model with the free surface present. Therefore, this example nicely illustrates that computing a solution where the free surface is removed is not sufficient to predict all upgoing waves that occur even in this very simple model with only one interface in the sub-surface.

Finally, Figs 8 and 9 show the up- and downgoing, $P$ - and $S$ wave constituents (vertical particle velocity) at the two recording levels 5 and $50 \mathrm{~m}$ below the free surface where fourth-order accurate FD approximations to eqs (2) and (3) were used to separate up- and downgoing wavefields into their respective $P$ - and $S$-wave constituents. It is now entirely straightforward to identify 

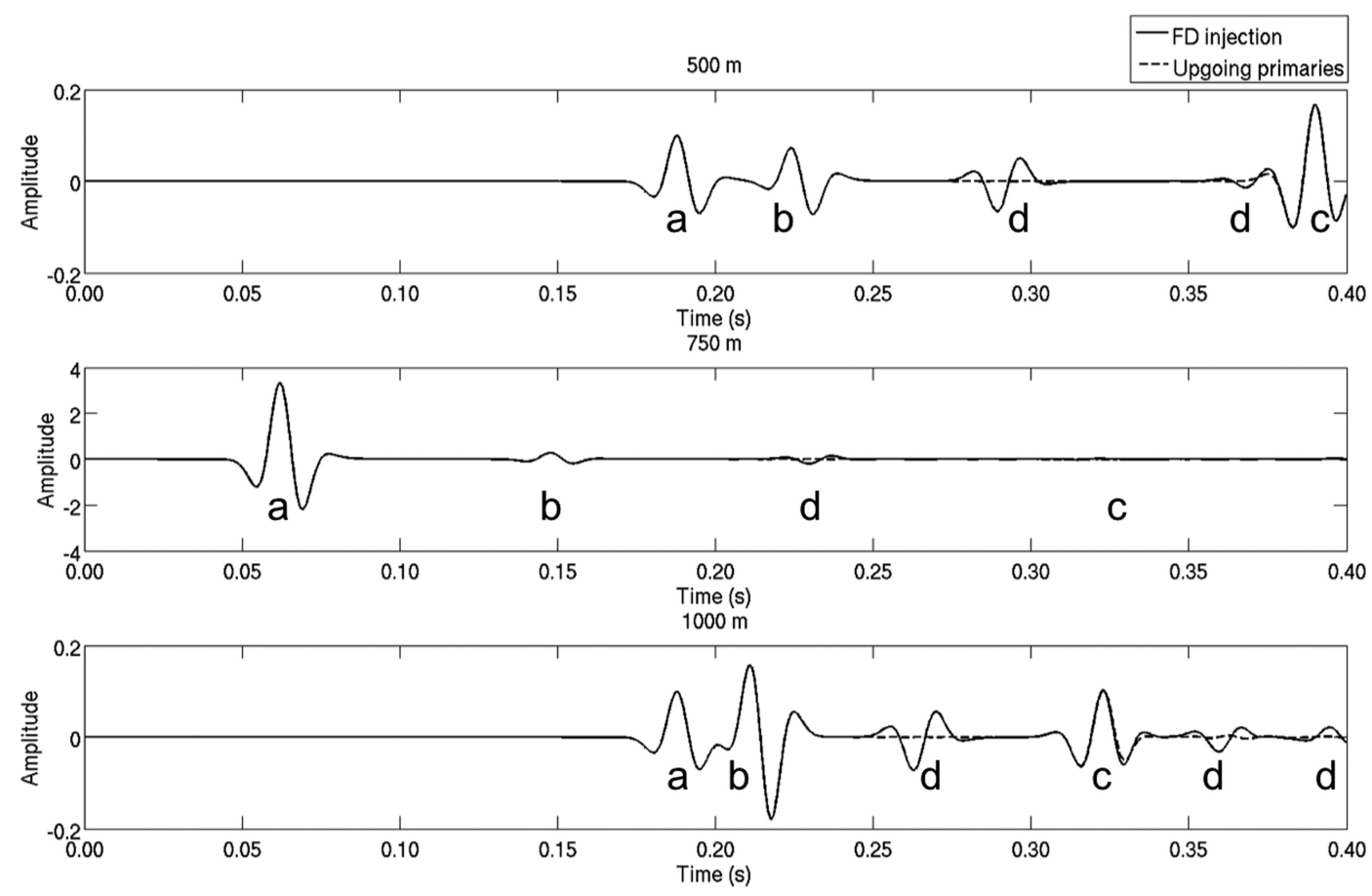

Figure 7. Comparison of upgoing wavefield constituent $50 \mathrm{~m}$ below free surface against reference solution (FD solution without a free surface present) at horizontal coordinate 500, 750 and $1000 \mathrm{~m}$. Annotated events: (a) direct $P$ wave, (b) $P P$ reflection from the interface, (c) $P S$ reflection from the interface, (d) free surface multiples.
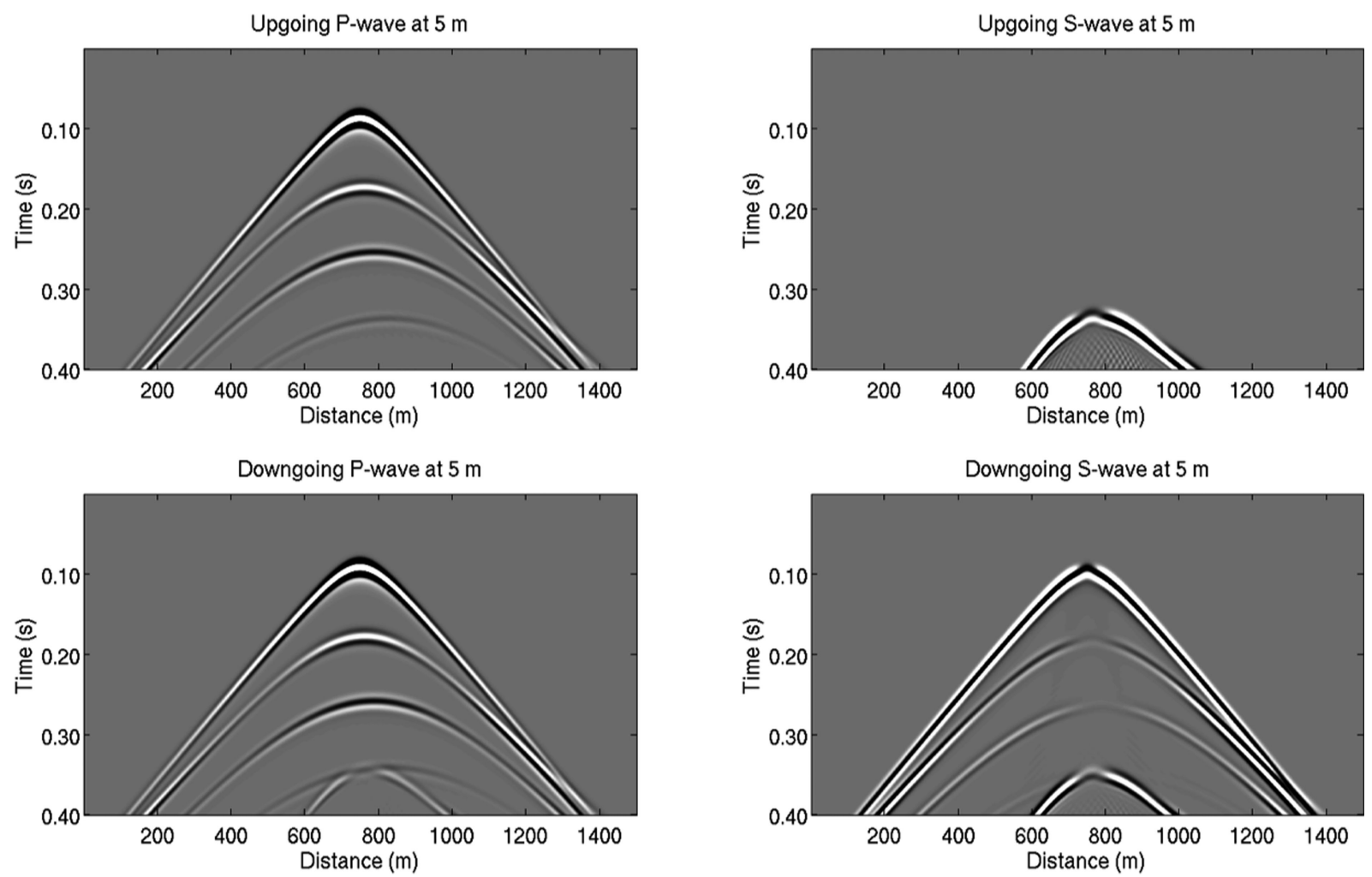

Figure 8. Up- and downgoing, $P$ - and $S$-wave constituents (vertical particle velocity) $5 \mathrm{~m}$ below the free surface. Top left: upgoing $P$. Top right: upgoing $S$. Bottom left: downgoing $P$. Bottom right: downgoing $S$.

the direct wave, reflections and conversions from the free surface, reflections and conversions from the dipping interface and multiples within the layer. Such results are essential for the development of seismic data processing techniques for instance for deghosting, multiple attenuation and noise attenuation. Although the model used here to demonstrate the methodology is simple, equivalent results are completely straightforward to produce for more complex models with strong vertical and lateral heterogeneity, at least for 

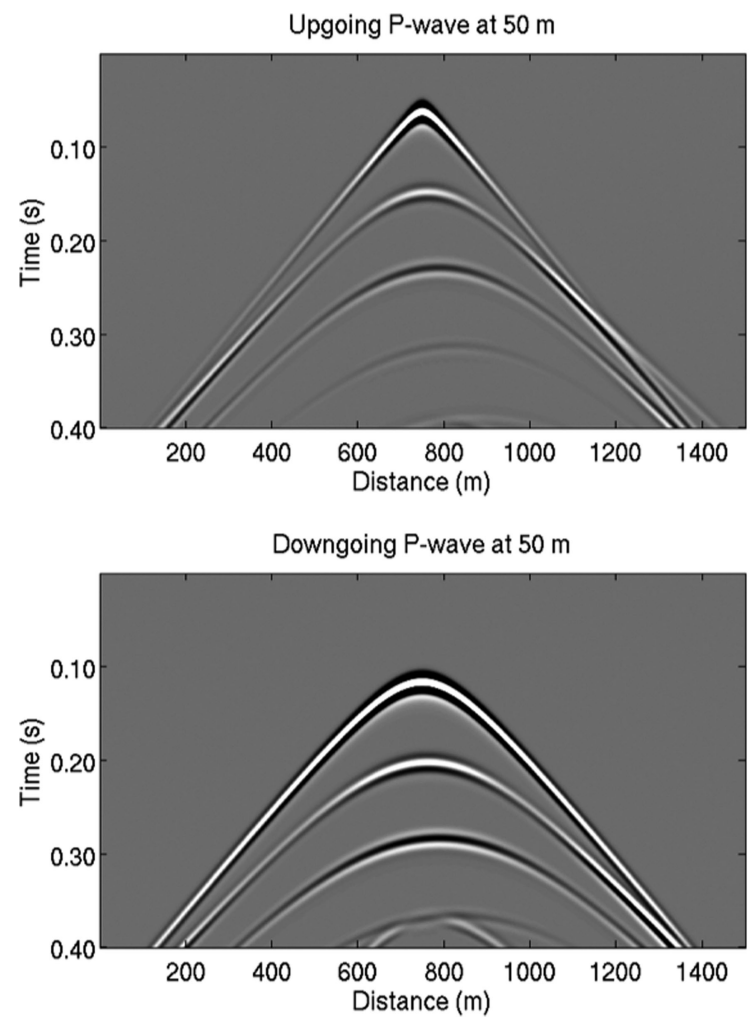
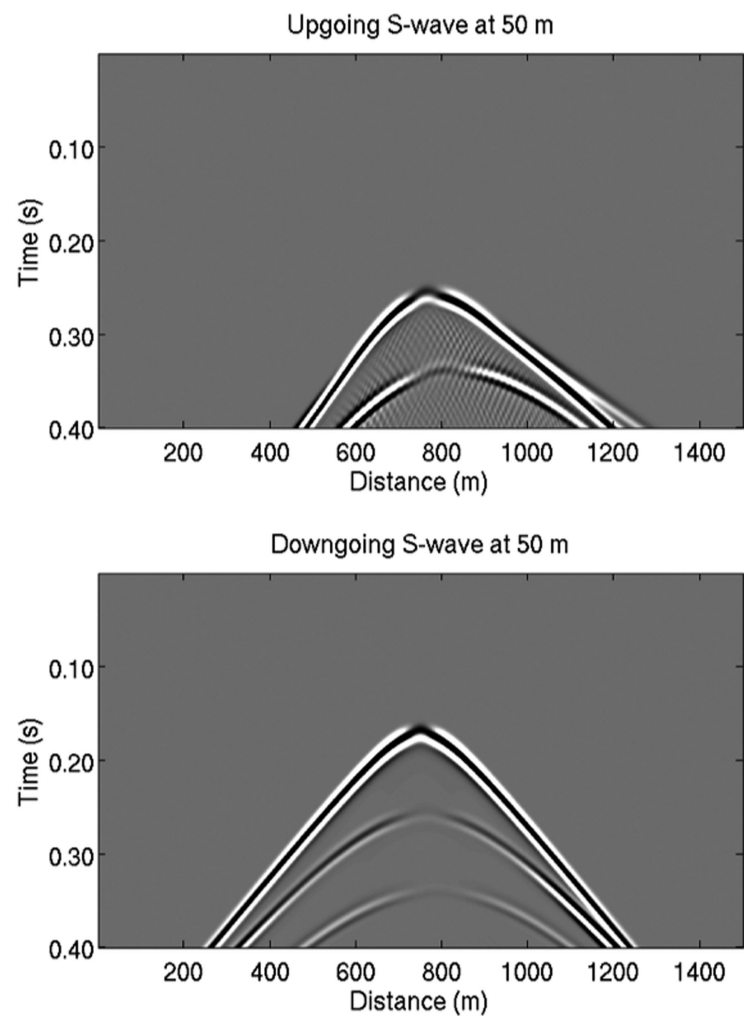

Figure 9. Up- and downgoing, $P$ - and $S$-wave constituents (vertical particle velocity) $50 \mathrm{~m}$ below the free surface. Top left: upgoing $P$. Top right: upgoing $S$. Bottom left: downgoing $P$. Bottom right: downgoing $S$.

cases when strong medium variations do not intersect the recording datum.

\section{DISCUSSION}

In this paper, we limited our discussion to the case where the material properties at the recording datum are homogeneous. The basic underlying principles of FD-injection are valid for arbitrarily complex media at the injection boundary. However, the problem of how to proceed occurs in the second step when injecting the recorded wavefield on the smaller truncated model to separate up- and downgoing wavefields. It is essential that injected waves on this model do not scatter from model heterogeneities so that the direction of propagation is altered. We expect that the methodology will work reasonably well also in mildly heterogeneous media when material properties are extended from the recording datum vertically above and below in the new truncated model. Although this is not entirely correct as the corresponding part of the full model may differ from such a structure, the results may be sufficiently accurate for many practical applications. However, it is clear that we no longer can argue that the results are accurate to within machine precision. There is also a more fundamental concern related to the underlying ambiguity as to what constitutes up- and downgoing $P$ and $S$ waves in media where the local propagation regime is considerably more complex (Weston 1989).

Some care must be taken when decomposing and recording the separate wavefield constituents to ensure that the output exactly corresponds to the desired depth level. The FD-injection based wavefield separation method described by Amundsen \& Robertsson (2014) injects upgoing waves upwards and downgo- ing waves downwards in the homogeneous medium during the wavefield separation computation. Therefore, depending on what distance that the wavefield is recorded from the injection surface, the upgoing wavefield is automatically redatumed upwards (or downwards for the downgoing waves) in the homogeneous model by a distance corresponding to the distance between the injection surface and the final output location. This does not represent a problem as long as it has been accounted for when identifying the location of the recording/injection surface in the original model.

Another aspect that needs to be considered is that only upgoing waves are injected upwards (and downgoing waves downwards). Since the total wavefield is known from the original simulation, both up- and downgoing waves can be computed even if only one part of them is computed during the wavefield separation computation. The other constituent is simply obtained by subtracting the computed wavefield constituent from the total wavefield.

Finally, as an alternative, we note that by reversing the wavefields in time and flipping polarity on particle motion data, it is possible to radiate downgoing waves upwards and upgoing waves downwards in the wavefield separation computation through reverse time extrapolation instead of the conventional forward time extrapolation during the FD computation.

\section{CONCLUSIONS}

We have presented a methodology for isolating up- and downgoing waves as well as $P$ and $S$ waves in conventional FD modelling. Isolating such wavefield constituents is required in many applications of modelling and less general modelling methods, such as the 
reflectivity method (Kennett 1983), have previously been the only option available.

Our methodology is based on the wavefield separation method presented by Amundsen \& Robertsson (2014) and relies on the FDinjection technique introduced by Robertsson \& Chapman (2000). In principle, the method is highly accurate (to within machine precision for up/down separation). However, other sources of noise such as reflections from absorbing boundaries or the degree of accuracy of the chosen $P / S$ separation operators are likely to dominate the error in the final result.

The method relies on one additional FD simulation to separate the data into up- and downgoing waves. However, for most applications the size of the required model for the additional simulation will be much smaller than that of the full FD simulation. The computational cost is therefore only slightly greater than that of a conventional FD simulation to generate synthetic data.

Finally, the methodology described is general and applies to different wave equations. In addition to the elastic isotropic case described in this paper, a similar methodology can be applied to anisotropic elastic as well as electromagnetic wave propagation problems for instance.

\section{ACKNOWLEDGEMENTS}

We wish to thank the CARNEVAL consortium sponsors Nagra, OMV and Schlumberger Gould Research for partial support of two of the co-authors of this paper. CVR's PhD research is supported through Swiss National Science Foundation grant 200021_156996. We thank editor Prof Jeannot Trampert, Dr Ivan Vasconcelos and one anonymous reviewer for comments that greatly helped improving the manuscript.

\section{REFERENCES}

Alterman, Z. \& Karal, F.C., 1968. Propagation of elastic waves in layered media by finite difference methods, Bull. seism. Soc. Am., 58, 367-398.

Amundsen, L., 2001. Elimination of free-surface related multiples without need of the source wavelet, Geophysics, 66, 327-341.

Amundsen, L., Ikelle, L.T. \& Martin, J., 2000. Multiple attenuation and P/S splitting of multicomponent $\mathrm{OBC}$ data at a heterogeneous sea floor, Wave Motion, 32, 67-78.

Amundsen, L. \& Robertsson, J.O.A., 2014. Prediction of wavefield constituents by modeling, Part I: Wave equation processing and imaging of marine multicomponent data beyond traditional RTM, Geophysics, 79, T287-T300.

Blanch, J.O., 2012. Is de-ghosting necessary with multi-component data?, Expanded abstract at the 84th SEG Annual Meeting.

Borisov, D. \& Singh, S.C., 2013. An efficient 3D elastic full waveform inversion of time-lapse seismic data using grid injection method, in 2013 SEG Annual Meeting, Society of Exploration Geophysicists.

Brenders, A.J. \& Pratt, R.G., 2007. Full waveform tomography for lithospheric imaging: results from a blind test in a realistic crustal model, Geophys. J. Int., 168, 133-151.

Chapman, C., 2004. Fundamentals of Seismic Wave Propagation, Cambridge Univ. Press.
Dellinger, J. \& Etgen, J., 1990. Wave-field separation in two-dimensional anisotropic media (short note), Geophysics, 55, 914-919.

Dragoset, W.H. \& Jericevic, Z., 1998. Some remarks on surface multiple attenuation, Geophysics, 63, 772-789.

Fokkema, J.T. \& van den Berg, P.M., 1993. Seismic Applications of Acoustic Reciprocity, Elsevier Science.

Halliday, D.F., Curtis, A., Vermeer, P., Strobbia, C., Glushchenko, A., van Manen, D.J. \& Robertsson, J.O.A., 2010. Interferometric ground-roll removal: Attenuation of scattered surface waves in single-sensor data, Geophysics, 75, SA15-SA25.

Kennett, B.L., 1983. Seismic Wave Propagation in Stratified Media, Cambridge Univ. Press.

Komatitsch, D. \& Martin, R., 2007. An unsplit convolutional perfectly matched layer improved at grazing incidence for the seismic wave equation, Geophysics, 72, SM155-SM167.

Levander, A.R., 1988. Fourth-order finite-difference P-SV seismograms, Geophysics, 53, 1425-1436.

Masson, Y., Cupillard, P., Capdeville, Y. \& Romanowicz, B., 2014. On the numerical implementation of time-reversal mirrors for tomographic imaging, Geophys. J. Int., 196, 1580-1599.

Moczo, P., Robertsson, J.O.A. \& Eisner, L., 2007. The finite-difference timedomain method for modelling of seismic wave propagation, Advances in Geophysics, 48, 421-516.

Özbek, A., Vassallo, M., Özdemir, K., van Manen, D.J. \& Eggenberger, K. 2010. Crossline wavefield reconstruction from multicomponent streamer data: Part 2-Joint interpolation and 3D up/down separation by generalized matching pursuit, Geophysics, 75, WB69-WB85.

Pletcher, R.H., Tannehill, J.C. \& Anderson, D., 2012. Computational Fluid Mechanics and Heat Transfer, CRC Press.

Ravasi, M. \& Curtis, A., 2013. Elastic imaging with exact wavefield extrapolation for application to ocean-bottom 4C seismic data, Geophysics, 78, S265-S284.

Regone, C.J., 2007. Using 3D finite-difference modeling to design wideazimuth surveys for improved subsalt imaging, Geophysics, 72, SM231SM239.

Robertsson, J.O.A. \& Amundsen, L., 2014. Wave equation processing using finite-difference propagators, Part 2. Deghosting of marine hydrophone seismic data, Geophysics, 79, Т301-T312.

Robertsson, J.O.A. \& Blanch, J.O., 2014. Numerical methods, finite difference, in Encyclopaedia of Solid Earth Geophysics (ESEG), 2nd edn, pp. 883-892, ed. Gupta, H., Springer Verlag.

Robertsson, J.O.A. \& Chapman, C.H., 2000. An efficient method for calculating finite-difference seismograms after model alterations, Geophysics, 65, 907-918.

Robertsson, J.O.A., Ryan-Grigor, S., Sayers, C. \& Chapman, C.H., 2000. A finite-difference injection approach to modeling of seismic fluid flow monitoring, Geophysics, 65, 896-906.

Vasconcelos, I., 2013. Source-receiver, reverse-time imaging of dualsource, vector-acoustic seismic data, Geophysics, 78, WA123WA145.

Virieux, J., 1986, $P-S$ V wave propagation in heterogeneous media: velocitystress finite-difference method, Geophysics, 51, 889-901.

Weston, V.H., 1989. Wave splitting and the reflection operator for the wave equation in R3, J. Math. Phys., 30, 2545-2562.

Yan, J. \& Sava, P., 2008. Isotropic angle-domain elastic reverse-time migration, Geophysics, 73, S229-S239.

Yan, J. \& Sava, P., 2009. Elastic wave-mode separation for VTI media, Geophysics, 74, WB19-WB32. 